\title{
Resonance Theory of Vibrational Strong Couplings in Polariton Chemistry
}

\author{
Xinyang Li, ${ }^{1}$ Arkajit Mandal,,${ }^{1, *}$ and Pengfei Huo ${ }^{1, \dagger}$ \\ ${ }^{1}$ Department of Chemistry, University of Rochester, Rochester, NY 14627
}

(Dated: September 3, 2020)

\begin{abstract}
In this work, we present a new theoretical explanation of the resonance vibrational strong coupling (VSC) regime in polariton chemistry. Coupling molecular vibrations and the cavity photonic excitation has experimentally demonstrated to strongly influence the ground state kinetics of a chemical reaction. Our theoretical results suggest that the VSC kinetics modification originates from the non-Markovian behavior of the cavity radiation mode when coupling to the molecule, leading to the dynamical caging of the reaction coordinate and the suppression of chemical reaction rate for a given range of photon frequency that is close to the barrier frequency. Further, we use a simple analytical non-Markovian rate theory to describe a single molecular system coupled to a radiation mode in an optical cavity. We demonstrate the accuracy of the rate theory by performing a numerical calculation in a one-dimensional model molecular system coupled to the cavity. Our simulations and analytical theory demonstrate the importance of dynamical effects in VSC polaritonic chemistry.
\end{abstract}

Introduction. Polariton Chemistry is an exciting emerging field [1-5] that provides opportunities for new chemical reactivities or selectivities by coupling molecular systems to quantized radiation fields inside an optical cavity. By hybridizing electronic excitation of the molecule and the photonic excitation of the radiation inside the cavity, new light-matter entangled states, socalled the polariton states, are generated with an energy gap, which is the Rabi splitting that indicate the coupling strength. Recent experimental and theoretical works have demonstrated the possibility of changing photo-isomerization reactivities [3, 6-9], modifying electron transfer kinetics [10-12], and remotely controlling chemical reactions [13]. These new polaritonic photochemical reactivities are attributed to the modification the excited state landscape $[1,3,6-9,11,12]$ due to the formation of the polariton states.

Similarly, hybridizing molecular vibrations and the photonic excitations inside an optical cavity [14, 15] forms vibrational polaritons. For the vibrational polaritonic hybrid system, it is a well-known result that the Rabi splitting observed in the infrared (IR) spectrum (due to light-matter coupling) scales as $\sqrt{N}$ with $N$ as the number of molecules $[14,15]$ inside the cavity. Whether or not such a collective effect also manifests itself into chemical kinetics has been a subject of a debate [16-19]. Recent experiments have demonstrated that it is possible to suppress [20-24] or enhance [25, 26] the ground state chemical reactivities by placing an ensemble of molecules in an optical micro-cavity through a resonance coupling between the cavity frequency and vibrational degrees of freedom. This so-called vibrational strong coupling (VSC) regime [5] operate in the absence of any light source (photons inside the cavity) [21, 22], and was hypothesized to utilize the hybridization of a vibrational transition of a molecule and the zero-point energy fluctuations of a cavity mode [21, 22]. This new strategy of VSC will allow us to bypass some intrinsic difficulties (such as intramolecular vibrational energy transfer) encountered in mode-selective chemistry that uses IR excitation to tune chemical reactivities, offering a paradigm-shift of synthetic chemistry through cavity enabled bond-selective chemical transformations [21, 22].

Unfortunately, a clear theoretical explanation of such remarkable VSC ground state reactivities remains missing, including explaining both (i) the collective $(N$ dependent) effects on chemical reaction rates, and (ii) the resonant effect where the suppression of the rate is achieved with a particular cavity photon frequency. Recent theoretical works that use simple transition state theory (TST) suggest that there is no collective effect nor resonant effect in VSC polariton chemistry [17-19, 27]. On the other hand, both effects do show up in a VSC non-adiabatic electron transfer reaction [28], with an enhancement of the rate upon resonance coupling between molecular vibration and the cavity. The applicability of this theory on the recent VSC ground state reactions remains an open question.

In this work, we provide a completely new perspective on understanding the resonance effect of the VSC ground state reactivities. Through both analytical theory and numerical simulations, we demonstrate that the non-Markovian nature of a radiation mode leads to significant suppression of the chemical reaction rate constant at a particular photon frequency that is related to the barrier frequency. At such a "resonance" frequency, the cavity radiation mode induces a dynamical-caging effect $[29,30]$, such that the molecular reaction coordinate becomes trapped in a narrow "photonic solvent cage" near the barrier region, leading to a suppression of the chemical kinetics. Such effects are purely dynamical and are not captured within a simple transition state theory. This work underscores the importance of "dynamical solvent effect" of the cavity radiation modes and provides a completely new understanding of the VSC polariton chemistry, paving the way towards an ultimate theoretical understanding of VSC polariton chemistry. 


\section{RESULT.}

Theoretical Model. The model QED Hamiltonian used in this work for describing the molecule-cavity interaction is expressed as [31-33]

$\hat{H}=\frac{\hat{P}^{2}}{2 M}+E(R)+\hat{H}_{\mathrm{vib}}+\frac{\hat{p}_{\mathrm{c}}^{2}}{2}+\frac{1}{2} \omega_{\mathrm{c}}^{2}\left(\hat{q}_{\mathrm{c}}+\sqrt{\frac{2}{\hbar \omega_{\mathrm{c}}^{3}}} \chi \cdot \mu(R)\right)^{2}$,

which is the Pauli-Fierz (PF) QED Hamiltonian (see Method) projected on the electronic ground state $\left|\Psi_{g}(R)\right\rangle$. Here, $E(R)$ is the ground state potential energy surface for a Shin-Metiu model (an electron and a proton confined between two fixed charged ions) depicted in Fig. 1b, where $R$ is a proton transfer coordinate, $\mu(R)=\left\langle\Psi_{g}|\hat{\mu}| \Psi_{g}\right\rangle$ is the ground state permanent dipole moment depicted in Fig. 1c, with $\hat{\mu}$ as the total dipole operator of the molecule. In addition, $\hat{H}_{\mathrm{vib}}=$ $\sum_{k} \frac{P_{k}^{2}}{2 M_{k}}+\frac{1}{2} M_{k} \omega_{k}^{2}\left(R_{k}+\frac{c_{k}}{M \omega_{k}^{2}} \cdot R\right)^{2}$ is the vibrational system-bath Hamiltonian that describes the interaction between reaction coordinate $R$ and other vibrational modes in the molecule. Further, $\hat{q}_{\mathrm{c}}=\sqrt{\hbar / 2 \omega_{\mathrm{c}}}\left(\hat{a}^{\dagger}+\hat{a}\right)$ and $\hat{p}_{\mathrm{c}}=i \sqrt{\hbar \omega_{\mathrm{c}} / 2}\left(\hat{a}^{\dagger}-\hat{a}\right)$ are the photonic coordinate and momentum operator, respectively, where $\hat{a}^{\dagger}$ and $\hat{a}$ are the photon creation and annihilation operators. Under the dipole gauge, the matter interact with the quantized radiation mode of the cavity by displacing the photonic coordinate with the amount of $\sqrt{\frac{2}{\hbar \omega_{c}^{3}}} \chi \cdot \mu_{0}(R)$, where $\chi$ characterizes the coupling strength between the molecule and the cavity (see Method). In this study, we have also explicitly assumed that the dipole moment is always aligned with the cavity polarization direction.

Vibrational Polariton Rabi Splitting. At the equilibrium position of the reactant $R_{0}$, one can approximate the permanent dipole as $\mu(R) \approx \mu_{0}+\mu_{0}^{\prime}\left(R-R_{0}\right)$, where $\mu_{0}=\mu\left(R_{0}\right)$ and $\mu_{0}^{\prime}=\left.\frac{\partial \mu(R)}{\partial R}\right|_{R_{0}}$. The light-matter interaction term in $\hat{H}$ (Eq. 1) at $R_{0}$ becomes [15, 17] $\sqrt{\frac{2 \omega_{\mathrm{c}}}{\hbar}} \hat{q}_{\mathrm{c}} \chi \cdot \mu\left(R_{0}\right)=\sqrt{\frac{\hbar}{2 M \omega_{0}}} \chi \cdot \mu_{0}^{\prime}\left(\hat{a}^{\dagger}+\hat{a}\right)\left(\hat{b}^{\dagger}+\hat{b}\right)+$ $\sqrt{\frac{2 \omega_{\mathrm{c}}}{\hbar}} \hat{q}_{\mathrm{c}} \chi\left(\mu_{0}-\mu_{0}^{\prime} R_{0}\right)$, where $\omega_{0}=\left.\frac{\partial E^{2}(R)}{\partial R^{2}}\right|_{R_{0}}$ is the vibrational frequency at the equilibrium nuclear configuration $R_{0}, M$ is the effective mass of the nuclear vibration, $\hat{b}^{\dagger}$ and $\hat{b}$ are the creation and annihilation operators for the nuclear vibration associated with the coordinate $R$. At the resonance condition of $\omega_{\mathrm{c}}=\omega_{0}$, the photon-vibration coupling induces a Rabi splitting $\hbar \Omega_{R}$ as follows $[15,17]$

$$
\hbar \Omega_{R}=2 \sqrt{\frac{\hbar}{2 M \omega_{0}}} \chi \cdot \mu_{0}^{\prime} \equiv 2 \hbar \omega_{\mathrm{c}} \cdot \eta
$$

where the normalized coupling strength $\eta=$ $\mu_{0}^{\prime} \sqrt{\frac{\hbar}{2 M \omega_{0}}} \frac{\chi}{\hbar \omega_{c}}$ characterizes the light-matter coupling strength. Note that the above relation between $\Omega_{R}$ and $\eta$ only holds under the linear approximation of (a)


FIG. 1. Vibrational strong coupling (VSC) regime in polariton chemistry. (a) Schematic representation of a molecule placed inside an optical cavity. (b) Ground state potential energy surface (PES) of the molecule as a function of the mass-weighted proton coordinate $\sqrt{M} R$ for the Shin-Metiu molecular model system. The ground state electronic density at two different nuclear configurations (at the donor and acceptor minima) are illustrated in the insets. (c) Ground state permanent dipole (solid red line) as a function of the mass weighted proton coordinate $\sqrt{M} R$. (d) Cavity BornOppenheimer (CBO) surface along photonic coordinates $q_{\mathrm{c}}$ and mass-weighted reaction coordinate $\sqrt{M} R$, with the white dash line representing the minimum energy path at the resonance frequency $\omega_{0}=\hbar \omega_{\mathrm{c}}=0.17$ $\mathrm{eV}$ and with a coupling strength $\eta=0.047$. (e) A zoomin to the reactant well of the CBO surface at the resonance frequency $\hbar \omega_{\mathrm{c}}=0.17$ and $\eta=0.376$. The arrows in (d)-(e) represent the directions of two polariton normal modes. (f) Schematic diagram showing the Rabisplitting $\hbar \Omega_{\mathrm{R}}$ due to the light-matter coupling between photon-dressed vibronic-Fock states, $\left|\nu_{0}, 1\right\rangle$ (photonic excitation) and $\left|\nu_{1}, 0\right\rangle$ (vibrational excitation). 
the dipole operator, and it breaks down for ultra-strong coupling (USC) regime when $0.1<\eta<1$ [34]. The $\hbar \Omega_{R}$ presented in the main text are instead obtained from numerical simulations of $\hat{H}$ (Eq. 1), with details provided in the Supporting Information.

Reaction Rate Constant. The VSC polariton chemical kinetics can be viewed as a barrier crossing process on the cavity Born-Oppenheimer surface (CBO) [17, $31,35] \hat{H}_{\mathrm{pl}}=E(R)+\hat{H}_{\mathrm{vib}}+\frac{1}{2} \omega_{\mathrm{c}}^{2}\left(\hat{q}+\sqrt{\frac{2}{\hbar \omega_{\mathrm{c}}^{3}}} \chi \cdot \mu(R)\right)^{2}$, which is a function of both $q_{\mathrm{c}}$ and $R$. Note that the correct QED description in Eq. 1 includes the dipole selfenergy $(\chi \cdot \mu(R))^{2} / \hbar \omega_{\mathrm{c}}$ (see method). Without this term, one would get artificial changes of the barrier height and predicts a significant modification of the polariton potential energy barrier [17]. Since we are interested in the VSC regime, the cavity mode has a similar range of frequency as the molecular vibrations, meaning that $q_{\mathrm{c}}$ evolve at a similar time scale as $R$. Based upon this consideration, we decide to follow the previous work [1719] to treat both nuclear and photonic DOF classically. The electronic DOF is considered fully quantum mechanically, described by the adiabatic electronically ground state wavefunction $\left|\Psi_{g}(R)\right\rangle$.

It is formally rigorous to express the rate constant as the TST rate $k_{\mathrm{TST}}$ and the transmission coefficient $\kappa$ as follows

$$
k=\lim _{t \rightarrow t_{\mathrm{p}}} \kappa(t) \cdot k_{\mathrm{TST}},
$$

where $t_{\mathrm{p}}$ refers to the plateau time of the flux-side correlation function, and $\kappa(t)$ is the transmission coefficient that captures the dynamical recrossing effects, measuring the ratio between the reaction rate and the TST rate. It has been shown that classically the potential mean force is invariant to the change the coupling strength or photon frequency [18], and other theoretical investigations based on a TST analysis for $N$ molecules coupled to cavity also suggest no significant change of the reaction rate [19, 27]. Since $k_{\text {TST }}$ does not change under the VSC condition, it is reasonable to conjecture that the change is purely dynamical and completely irrelevant to the potential barrier changes or free energy barrier changes. Thus, it is highly likely that such influence of the cavity on VSC chemical reactivities is purely originated from the transmission coefficient $\kappa$. It can be numerically calculated from the flux-side correlation function formalism [36, 37] as follows

$$
\kappa(t)=\frac{\left\langle\mathcal{F}(0) \cdot h\left[R(t)-R_{\ddagger}\right]\right\rangle_{\ddagger}}{\left\langle\mathcal{F}(0) \cdot h\left[\dot{R}_{\ddagger}(0)\right]\right\rangle_{\ddagger}},
$$

where $h\left[R-R_{\ddagger}\right]$ is the Heaviside function of the reaction coordinate $R$, with the dividing surface $R_{\ddagger}$ that separate the reactant and the product regions (for the particular model studied here, $R_{\ddagger}=0$ ), the flux function $\mathcal{F}(t)=\dot{h}(t)=\delta\left[R(t)-R_{\ddagger}\right] \cdot \dot{R}(t)$ measures the reactive flux across the dividing surface (with $\delta(R)$ as the Dirac delta function), and $\langle\ldots\rangle_{\ddagger}$ represents the ensemble average on the dividing surface where the reaction coordinate $R$ is constrained with the value $R_{\ddagger}$.

To obtain more intuitive understanding of how VSC light-matter interaction can influence $\kappa$, let us consider a simplified model, $\hat{H}-\hat{H}_{\text {vib }}$ where we only have two DOFs $\left\{R, q_{\mathrm{c}}\right\}$ such that we can obtain an analytic expression of the rate as $k=k_{\mathrm{TST}} \cdot \kappa_{\mathrm{GH}}$, where the transmission coefficient $\kappa_{\mathrm{GH}}$ (under the limit $t \rightarrow t_{\mathrm{p}}$ ) can be obtained from the Grote-Hynes (GH) theory $[29,38-42]$. The TST rate is $k_{\mathrm{TST}}=\frac{\omega_{0}}{2 \pi} e^{-\beta E_{\mathrm{b}}}$, where $E_{\mathrm{b}}=E\left(R_{\ddagger}\right)-E\left(R_{0}\right)$ is the potential energy barrier height measured from the bottom of the well $R_{0}$ to the top of the barrier $R_{\ddagger}$ (see Fig. 1), and $\omega_{0}$ is the vibrational frequency of the reactant at $R=R_{0}$, and $\beta=\left(k_{\mathrm{B}} T\right)^{-1}$. When explicitly considering the DSE, $E_{\mathrm{b}}$ remains invariant as changing the lightmatter coupling strength or the photon frequency (see Eq. 1), explaining why one can not observe any effects from a simple TST analysis [18]. The transmission coefficients $\kappa_{\mathrm{GH}}$ for this simple 2D model is (see the Method section)

$$
\kappa_{\mathrm{GH}}=\frac{1}{\omega_{\mathrm{b}}}\left[\frac{1}{2}\left(-\Delta \omega_{\ddagger}^{2}+\sqrt{\left(\Delta \omega_{\ddagger}^{2}\right)^{2}+4 \omega_{\mathrm{b}}^{2} \omega_{\mathrm{c}}^{2}}\right)\right]^{\frac{1}{2}},
$$

where $\omega_{\mathrm{b}}^{2}=-\left.\frac{\partial^{2} E_{0}(R)}{\partial R^{2}}\right|_{R_{\ddagger}}$ is the curvature of the reaction barrier (and $\omega_{\mathrm{b}}$ is the barrier frequency), $\Delta \omega^{2} \equiv$ $\omega_{\mathrm{c}}^{2}-\omega_{\mathrm{b}}^{2}+\frac{\mathcal{C}_{\ddagger}^{2}}{\omega_{\mathrm{c}}^{2}}$, with $\mathcal{C}_{\ddagger}=\sqrt{\frac{2 \omega_{\mathrm{c}}}{M}} \chi \cdot \mu_{\ddagger}^{\prime}$ characterizes the effective coupling between photonic coordinate $q_{\mathrm{c}}$ and nuclear reaction coordinate $R$ in the transition state region, and $\mu_{\ddagger}^{\prime}=\left.\frac{\partial \mu}{\partial R}\right|_{R_{\ddagger}}$ is the slope of the dipole moment on the dividing surface $R_{\ddagger}$. Based on Eq. 5 , one can derive that $\kappa_{\mathrm{GH}}$ will have a minimum when

$$
\omega_{\mathrm{c}}=-\frac{1}{2} \tilde{\eta}^{2} \mu_{\ddagger}^{\prime 2}+\frac{1}{2} \sqrt{\tilde{\eta}^{4} \mu_{\ddagger}^{\prime 4}+4 \omega_{\mathrm{b}}^{2}}
$$

where $\tilde{\eta}=\frac{\chi}{\sqrt{M} \hbar \omega_{c}}$ (note that the normalized coupling strength is $\left.\eta=\mu_{0}^{\prime} \sqrt{\frac{\hbar}{2 \omega_{0}}} \tilde{\eta}\right)$. This provides a resonance effect of the reaction rate constant (through the transmission coefficient) when the cavity frequency $\omega_{c}$ is tuned. When $\eta$ is small (such that $\tilde{\eta}^{4} \mu_{\ddagger}^{\prime 4} \ll 4 \omega_{\mathrm{b}}^{2}$ ), the resonance frequency is close to the original barrier frequency $\omega_{\mathrm{b}}$. As the coupling strength $\eta$ increases, the minimum will be shifted to the low-frequency region (red-shift). Note that this resonance condition to achieve a minimum in $\kappa$ (Eq. 6) is different than the one (which is $\omega_{\mathrm{c}}=\omega_{0}$ ) to form the vibrational polariton in Eq. 2.

When explicitly considering the vibrational coupling to $R$ within $\hat{H}_{\text {vib }}, \kappa_{\mathrm{GH}}$ has a more complicated expression as shown in the Supporting Information. Nevertheless, the presence of $\hat{H}_{\text {vib }}$ does not change the resonance condition in Eq. 6 (see Fig. S1 in Supporting Information). The detailed procedure for obtaining the transmission coeffi- 

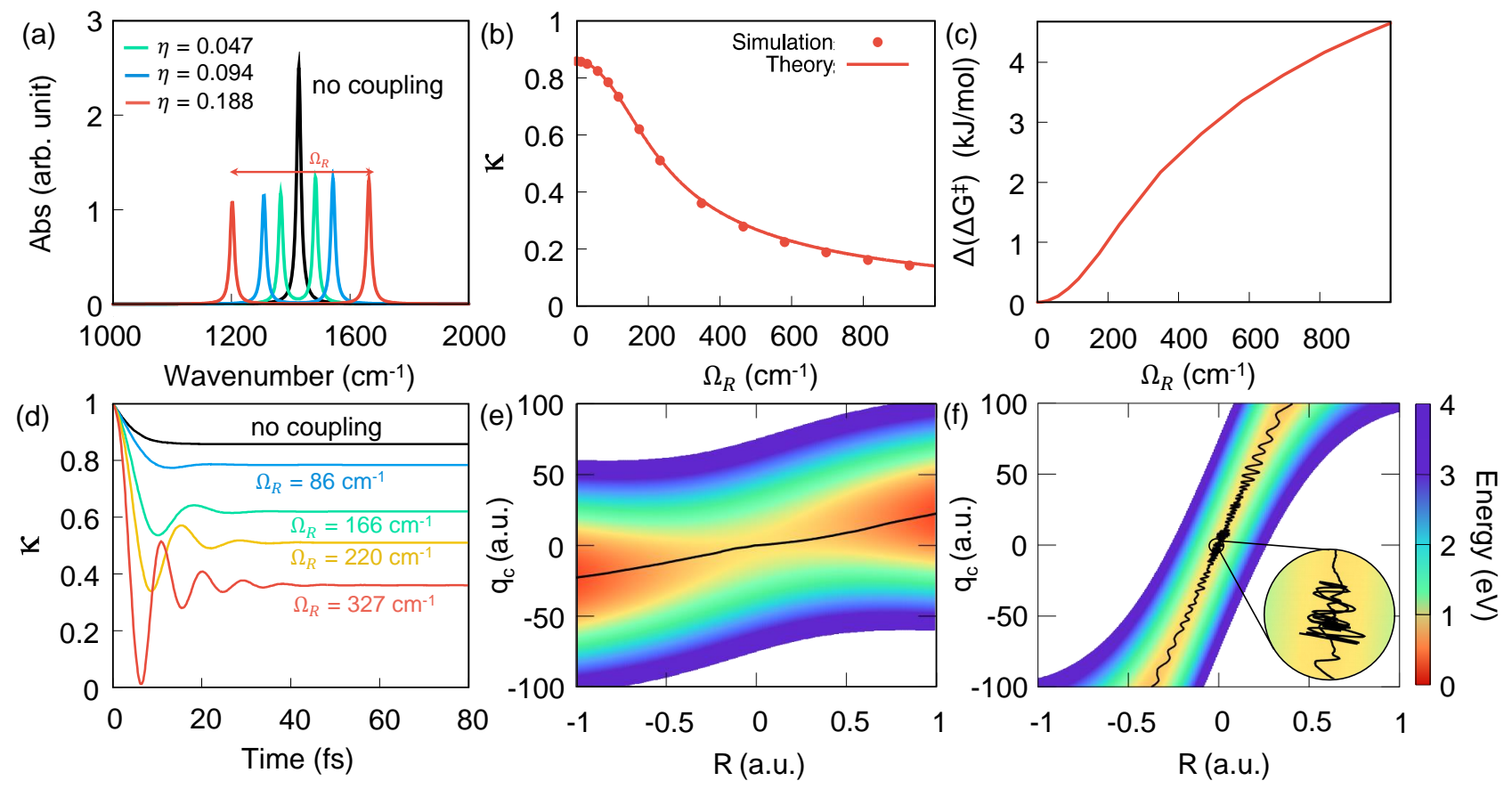

FIG. 2. Infrared absorption spectrum by changing the normalized light-matter coupling strength $\eta$ (see Eq 2). (b) The transmission coefficient $\kappa$ (under the limit $t \rightarrow t_{\mathrm{p}}$ ) under various light-matter coupling strength (indicated by $\Omega_{\mathrm{R}}$ ) at the resonance frequency $\omega_{\mathrm{c}}=\omega_{0}=0.17 \mathrm{eV}$. (c) The "effective change" of the Gibbs free energy barrier $\Delta\left(\Delta G^{\ddagger}\right)$ with respect to the coupling strength $\Omega_{\mathrm{R}}$ at $300 \mathrm{~K}$. (d) Time-dependent transmission coefficient $\kappa(t)$ at various light-matter coupling strengths. (e) and (f) Cavity Born-Oppenheimer surfaces $\hat{V}_{\mathrm{CBO}}\left(R, q_{\mathrm{c}}\right)$ at $\eta=0.047$ and $\eta=0.376$, respectively, with representative reactive trajectories indicated with black solid lines.

cient is provided in the method section as well as in the Supporting Information.

Central Hypothesis. With the above analysis, we conjecture that the quantized radiation mode inside the optical cavity is effectively acting as a "solvent" degree of freedom (DOF) that is coupled to the molecular reaction coordinate $R$, such that the presence of photonic coordinate enhance the recrossing of the reaction coordinate and reduces the transmission coefficients. A similar phenomenon is commonly referred to as the "dynamical caging" regime in simple organic reactions $[30,43,44]$ and enzymatic catalysis [45-47], which have been successfully explained by the GH theory.

Decreasing $\kappa$ as increasing $\Omega_{\mathrm{R}}$. Fig. 2 presents the influence of increasing light-matter coupling $\eta$ (thereby increasing $\Omega_{\mathrm{R}}$ ) on the reaction transmission coefficient $\kappa$ with the model Hamiltonian presented in Eq. 1. Fig. 2a presents the IR spectrum computed based the quantum light-matter interaction (Eq. 12 in Method). The numerically exact Rabi splitting $\hbar \Omega_{\mathrm{R}}$ is slightly deviated from $2 \hbar \omega_{\mathrm{c}} \cdot \eta$ (as indicated by Eq. 2) due to the linear approximation $\left(\mu(R) \approx \mu_{0}+\mu_{0}^{\prime} R\right.$ ) used in Eq. 2 (see Fig. S2 in the Supporting Information). Fig. 2b presents the transmission coefficient $\kappa$ obtained from direct numerical simulations (Eq. 4 under the $t \rightarrow t_{\mathrm{p}}$ limit) as well as from the GH theory (solid lines) $\kappa_{\mathrm{GH}}$ (by solving Eq. 10 in Method). The GH theory quantitatively agrees with the results from the direct numerical simulations. With an increasing Rabi splitting $\Omega_{R}$, the transmission coefficient $\kappa$ decreased by almost one order of magnitude, whereas the TST rate $k_{\mathrm{TST}}$ remains unchanged (due to the unchanged barrier height in the PF QED Hamiltonian). These numerical results corroborate our hypothesis that the suppression of chemical rate originates from $\kappa$, which closely resembles the experimental result (Fig. 3D in Ref. [22]).

Fig. 2c presents another interesting result in this work. For the PF Hamiltonian description that explicitly includes the DSE term, there is no change in $k_{\mathrm{TST}}$ because there is no change of potential energy barrier (see Fig. 1d) nor free energy barrier [18]. The only changes in the rate come from $\kappa$. However, one can back out the "effective change" of the free energy barrier height due to the changing $\kappa$. To this end, we use the Eyring Rate equation (Eq. 11 in Method) to convert the change of rate from $\kappa$ into an effective $\Delta\left(\Delta G^{\ddagger}\right)$. The 4 times decreases in $\kappa$ presented in Fig. $2 \mathrm{~b}$ results in $4 \mathrm{KJ} / \mathrm{mol}$ change in "effective $\Delta\left(\Delta G^{\ddagger}\right)$ in Fig. 2c over the $700 \mathrm{~cm}^{-1}$ of $\Omega_{\mathrm{R}}$. We emphasize that this is not the real change of the free energy barrier height, but rather an "effective" change of $\Delta G^{\ddagger}$ according to the change of $\kappa$ based on our theoretical analysis. Interestingly, the experimentally measured results of $\Delta\left(\Delta G^{\ddagger}\right)$ (Fig. 3C in Ref. 22, for example) closely resemble our theoretical finding in 
Fig. 2c, with the key difference that our theoretical investigate suggests that these are not the actual free energy barrier changes, but entirely due to the change of $\kappa$, i.e., kinetics. Note that if one hypothesizes that an unknown mechanism to force the upper or lower vibrational polariton states to be a gateway of VSC polaritonic chemical reaction [48], then the activation energy change should shift linearly [18] with $\Omega_{\mathrm{R}}$. The experimental results, on the other hand, demonstrate a non-linearity of reaction barrier [22]. Our theory indicates a non-linear increase of the "effective" $\Delta\left(\Delta G^{\ddagger}\right)$ as increasing $\Omega_{\mathrm{R}}$ due to the change of $\kappa$, closely resembles the experimental discoveries (Fig. 3c-d in Ref. [22]).

Fig. 2d presents the time-dependent simulation of the transmission coefficient $\kappa(t)$ defined in Eq. 4. With an increasing light-matter coupling hence a larger $\Omega_{R}$, the plateau value of $\kappa(t)$ keeps decreasing, and at the same time, becomes more oscillatory. This is typical behavior of reaction dynamics in the solvent caging regime [49]. As the coupling between $q_{\mathrm{c}}$ and $R$ increase, the non-Markovian dynamics of $q_{\mathrm{c}}$ can significantly influence the recrossing dynamics of the reaction coordinate $R$, from the "non-adiabatic" limit for a weak coupling regime to the "dynamic caging" for a strong coupling regime $[38,49]$.

To clearly demonstrate the difference between these two regimes, we further present the Cavity BO surface $\hat{V}_{\mathrm{CBO}}=\hat{H}-\frac{\hat{P}^{2}}{2 M}-\frac{\hat{p}_{\mathrm{c}}^{2}}{2}-\hat{H}_{\mathrm{vib}}$ along $R$ and $q_{\mathrm{c}}$ in panel (e) and (f), with a representative reactive trajectory on top (black solid line). Fig. 2e presents a typical non-adiabatic case of the GH theory. When the instantaneous friction is weak $\left(\frac{\mathcal{C}}{\omega_{c}} \ll \omega_{\mathrm{b}}\right)$, the GH theory becomes a model of non-equilibrium solvation, where the friction from the photonic coordinate $q_{\mathrm{c}}$ does not severely impede the transitions [49]. In this case, the transmission coefficient may remain close to those without the cavity (black curve in Fig. 2d), and the reactive trajectory crosses the barrier without much influence from $q_{\mathrm{c}}$. Fig. 2e presents a typical "dynamical caging" regime of the GH theory, where the instantaneous friction from $q_{\mathrm{c}}$ to $R$ is strong $\left(\frac{\mathcal{C}}{\omega_{\mathrm{c}}} \gg \omega_{\mathrm{b}}\right)$, such that the reaction coordinate $R$ becomes trapped in a narrow "solvent cage" at the barrier top [49]. At long times, the bath relaxation of $\hat{H}_{\text {vib }}$ allowing the $R$ to move away from the barrier top, but at shorter times, the reaction coordinate $R$ oscillates within the photon-induced solvent cage [50]. The trajectory cross the $R_{\ddagger}=0$ surface many times, resulting in oscillations of $\kappa(t)$ (and even exhibit negative values) at a short time and a small plateau value of $\kappa(t)$ at $t_{\mathrm{p}}$ (see red curve in Fig. 2d). This dynamical caging effects from the solvent have been extensively studied in simple organic reactions (SN1 and SN2) [30, 43, 44] and enzymatic reactions [45-47], where the solvent dynamics significantly influences the reaction rate $[38,39,49,51,52]$. Here, the photonic coordinate $q_{\mathrm{c}}$ acts like a "solvent coordinate", and for strong coupling between $q_{\mathrm{c}}$ and $R$, the system exhibits the dynamical caging effect, and effectively slows down the reaction rate constant. This is our theoretical explanation for the observed slow down of the rate constant for VSC polariton chemical reactions [20, 21, 23, 24].

The Origin of the Resonance Effect. Fig. 3a presents the transmission coefficient $\kappa$ (when $t \rightarrow t_{\mathrm{p}}$ ) as a function of the photon frequency $\omega_{\mathrm{c}}$ with three normalized coupling constant $\eta$ (defined in Eq. 2). The results are obtained from the GH theory (solid line) as well as the direct numerical simulation of Eq. 4 (filled circles). One can clearly see a resonance behavior of $\kappa$ when changing the photon frequency, agreeing with the analytical result (Eq. 6) of a simpler model. These findings in Fig. 3a closely resemble recent experimental results of desilylation reaction (Fig. 3A in Ref. 53, Fig. 3B in Ref. [20]), aldehyde/ketone Prins cyclization (Fig. 3 in Ref. [24]), and enzymatic reaction in pepsin (Fig. 3C in Ref. [23]). Note that under a relatively small light-matter coupling $\eta=0.047$ (green), the resonance frequency that gives a minimal $\kappa$ is close to $\omega_{\mathrm{b}}$, which is also close to the reactant equilibrium frequency of the reactant $\omega_{0}$ in our ShinMetiu model. For the parameter regime $\eta<0.1$ (not entering into the USC), we find that the resonance condition (based on Eq. 6) is close to $\omega_{\mathrm{b}}$. Note that experimentally, one often plot the cavity frequency-dependent reaction kinetics against the absorption curve of vibrational polariton. With our theoretical understanding and model calculations, we conclude that these two resonance behavior have two different origins and resonance frequencies. The resonance condition observed in the IR spectrum for Rabi Splitting requires $\omega_{\mathrm{c}}=\omega_{0}$, whereas the resonance effects for a minimum of the rate constant requires $\omega_{\mathrm{c}} \approx \omega_{\mathrm{b}}$. However, it is possible for a given molecular system which has $\omega_{0} \approx \omega_{\mathrm{b}}$.

When increasing the coupling strength to the USC regime $(0.1<\eta<1.0)$, the resonant frequency is significantly red-shifted from $\omega_{\mathrm{b}}$. For example, when $\eta=0.188$ (red curve), the resonant condition for reaching a minimum value of $\kappa$ is $25 \mathrm{meV}$. Nevertheless, in the range of $10 \mathrm{meV}<\omega_{\mathrm{c}}<100 \mathrm{meV}, \kappa$ remains a very low value around 0.2 , similar to the value at $\omega_{\mathrm{c}}=\omega_{\mathrm{b}}$. This red-shift of resonance frequency at which the rate is most significantly reduced has not been experimentally observed.

The origin of this resonant behavior in VSC chemical reaction rate constant can also be intuitively understood by examining representative trajectories on the cavity BO potential energy surfaces presented in Fig. 3b-d, with the black solid lines, indicate representative trajectories. At a very low frequency, $\hbar \omega_{\mathrm{c}}=2.5 \mathrm{meV}$ as shown in Fig. $3 \mathrm{~b}$, the photon coordinate essentially remains frozen compared to the dynamics of the reaction coordinate $R$ during the course of the reaction. As a result, under this frozen solvent limit, the transmission coefficient remains close to the no-coupling scenario. At $\hbar \omega_{\mathrm{c}}=80 \mathrm{meV}$ in Fig. 3c, with $\frac{\mathcal{C}}{\omega_{\mathrm{c}}} \gg \omega_{\mathrm{b}}$, the light-matter interactions leads 

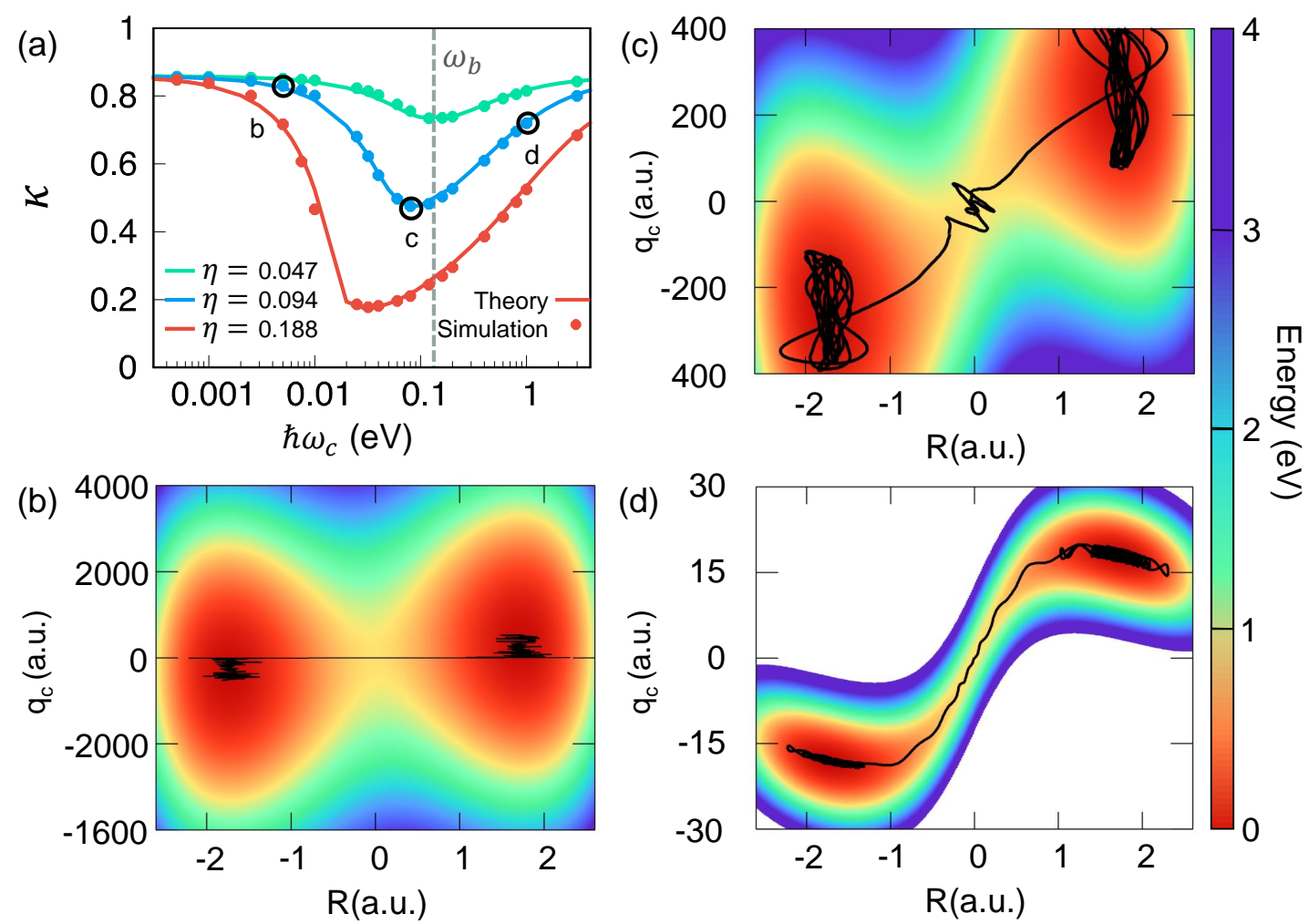

FIG. 3. Resonance effect in vibrational strong coupling regime of polariton chemistry. (a) Transmission coefficient $\kappa$ as a function of the photon frequency at three different coupling strength $\eta$. (b)-(d) presents the cavity Born-Oppenheimer surfaces $\hat{V}_{\mathrm{CBO}}\left(q_{\mathrm{c}}, R\right)$ under the normalized coupling strength $\eta=0.094$ (corresponding to the blue solid line in panel (a)) at the photon frequency (b) $\omega_{\mathrm{c}}=2.5 \mathrm{meV}$, (c) $80 \mathrm{meV}$, and (d) $1.0 \mathrm{eV}$, with the representative reactive trajectories indicated with black solid lines.

to the dynamical caging of the reaction coordinate at the barrier top leading to a significant decrease in the transmission coefficient $\kappa_{\mathrm{GH}}$. When the photon frequency is further increased, the reactant and the product wells become separated with a narrow channel as shown in Fig. 3d when $\hbar \omega_{c}=1 \mathrm{eV}$. At such a high photon frequency, the channel connecting the reactant becomes extremely narrow [54] (much narrower than the usual dynamical caging scenario depicted in Fig. 3c or Fig. 2f), such that the reactive trajectories almost follow a straight path and is no longer caged near the dividing surface. As opposed to the dynamic caging regime, the transmission coefficient in Fig. 3d is less suppressed than the minimum $\kappa$ when the photon frequency is near $\omega_{\mathrm{b}}$. Similar behavior of the reaction dynamics is also observed for the USC regime $(\eta=0.188$ in Fig. 3), where the results are provided in the Supporting Information. Therefore, the suppression of the chemical kinetics through the dynamical-caging effect by the photon mode is highly sensitive on the photon frequency, proving a plausible mechanism for explaining the resonance behavior $[21,23,24]$ of the reaction rate in VSC polariton chemistry.

Conclusions. In this work, we provide a new theoretical explanation of the resonance VSC polariton chem- istry reactivities. We demonstrate the resonant suppression of the reaction rate constant by using the analytical Grote-Hynes rate theory as well as performing numerical calculations for a Shin-Metiu model molecular system coupled to a single radiation mode inside an optical cavity. As opposed to the previous studies [17-19, 27] that only focuses on the transition state theory, our investigation suggests that coupling a cavity photonic mode to a molecule leads to the suppression of the transmission coefficient of the rate constant, which exhibit the resonance behavior that can be well explained by simple GH rate theory. Through both analytical theory and numerical simulations, we demonstrate that the cavity photon mode acts like a solvent coordinate which influences the chemical kinetics and leads to the suppression of the transmission coefficient. Such an effect is purely dynamical and is not captured within a simple transition state theory. This also explains why the polariton mediated reaction free energy barrier change is not necessarily linear to the Rabi splitting [5, 18, 22], because the former (if we back out the effective barrier change from the change of the rate constant) is a pure kinetic effect, which is not directly related to the latter, which is the energy splitting among vibrational polaritonic states 
in the reactant region.

Further, our theoretical hypothesis provides a plausible explanation to the observed resonance effects of the electronically adiabatic ground-state reactions coupled to an optical cavity, whereas previous theoretical studies [17-19,27] based upon a simple TST always conclude a frequency-independent VSC rate constant. The suppression of the rate constant is sensitive to the photon frequency, such that the maximum suppression is achieved when the photon frequency is close to the barrier frequency in the vibrationally strong coupling regime $\eta<0.1$ and is then red-shifted in the vibrationally ultrastrong coupling regime $0.1<\eta<1$. Our results indicate that the resonance condition for achieving the Rabisplitting in the IR-spectrum and the resonance condition for achieving a maximum suppression of the reaction rate constant are actually different. While the former is related to the frequency of the reactant, the latter is related to the barrier frequency and the coupling strength. Our work underscores the importance of the dynamical effect induced by the cavity photon modes on chemical kinetics to explain new chemical reactivities observed in recent experimental studies on vibrational strong coupling of molecules and cavity. Future investigations will focus on understanding the collective VSC reactivities by coupling many molecules with the cavity $[18,19]$.

\section{METHOD}

Pauli-Fierz QED Hamiltonian. The minimal coupling QED Hamiltonian in the Coulomb gauge (the "p $\cdot \mathrm{A}$ " form) is expressed as

$$
\hat{H}_{\mathrm{C}}=\sum_{j} \frac{1}{2 m_{j}}\left(\hat{\mathbf{p}}_{j}-z_{j} \hat{\mathbf{A}}\right)^{2}+\hat{V}(\hat{\mathbf{x}})+\hat{H}_{\mathrm{ph}},
$$

where the sum is performed over all charged particles, including electrons and nuclei, $m_{j}$ and $z_{j}$ are mass and charge for particle $j$,respectively, and $\hat{\mathbf{p}}_{j}=-i \hbar \boldsymbol{\nabla}_{j}$ is the canonical momentum operator. Further, under the Coulomb gauge, $\boldsymbol{\nabla} \cdot \hat{\mathbf{A}}=0$, the vector potential becomes purely transverse $\hat{\mathbf{A}}=\hat{\mathbf{A}}_{\perp}$. Under the long-wavelength approximation, $\hat{\mathbf{A}}=\mathbf{A}_{0}\left(\hat{a}+\hat{a}^{\dagger}\right)=\mathbf{A}_{0} \sqrt{2 \omega_{\mathrm{c}} / \hbar} \hat{q}_{\mathrm{c}}$, where $\mathbf{A}_{0}=\sqrt{\hbar / 2 \omega_{\mathrm{c}} \varepsilon_{0} \mathcal{V}} \hat{\mathbf{e}}$, with $\mathcal{V}$ as the quantization volume inside the cavity, $\varepsilon_{0}$ as the permittivity, and $\hat{\mathbf{e}}$ is the unit vector of the field polarization. Using the Power-ZienauWoolley (PZW) gauge transformation operator [55, 56] $\hat{U}=\exp \left[-\frac{i}{\hbar} \hat{\boldsymbol{\mu}} \cdot \hat{\mathbf{A}}\right]=\exp \left[-\frac{i}{\hbar} \hat{\boldsymbol{\mu}} \cdot \mathbf{A}_{0}\left(\hat{a}+\hat{a}^{\dagger}\right)\right]$, as well as a unitary transformation operator $\hat{U}_{\phi}=\exp \left[-i \frac{\pi}{2} \hat{a}^{\dagger} \hat{a}\right]$, the Pauli-Fierz (PF) Hamiltonian is obtained as

$\hat{H}_{\mathrm{PF}}=\hat{U}_{\phi} \hat{U} \hat{H}_{\mathrm{C}} \hat{U}^{\dagger} \hat{U}_{\phi}^{\dagger}=\hat{H}_{\mathrm{M}}+\frac{1}{2} \hat{p}_{\mathrm{c}}^{2}+\frac{1}{2} \omega_{\mathrm{c}}^{2}\left(\hat{q}_{\mathrm{c}}+\sqrt{\frac{2}{\hbar \omega_{\mathrm{c}}}} \hat{\boldsymbol{\mu}} \cdot \mathbf{A}_{\mathbf{0}}\right)^{2}$

where the matter Hamiltonian is $\hat{H}_{\mathrm{M}}=\hat{T}_{R}+\hat{H}_{\mathrm{el}} \equiv$ $\hat{T}_{R}+\hat{T}_{r}+\hat{V}$, with $\hat{T}_{R}$ and $\hat{T}_{r}$ representing the nuclear and electronic kinetic energy, respectively, and $\hat{V}$ representing the Coulomb interaction potential among all charged particles (electrons and nuclei), and $\hat{H}_{\mathrm{el}}$ is the electronic Hamiltonian. The detailed derivation is documented in the Supporting Information. The presence of dipole selfenergy (DSE) (the $A_{0}^{2}$ term in Eq. 7) is necessary in order to have a Gauge invariant Hamiltonian $[57,58]$ and it has shown to be crucial for an accurate description of lightmatter interactions under the dipole gauge [57-59]. Projecting the above Hamiltonian in the ground electronic state of the molecule $\left|\Psi_{g}\right\rangle$ (which is obtained by solving $\hat{H}_{\mathrm{el}}\left|\Psi_{g}\right\rangle=E_{g}(R)\left|\Psi_{g}\right\rangle$, we obtain the model Hamiltonian in Eq. 1.

Model Molecular Hamiltonian. The potential energy surface (PES) and permanent dipole moment are taken from a Shin-Metiu model [60], which is illustrated in Fig. 1. The Shin-Metiu model is an one dimensional molecular system that describes a proton-coupled electron transfer reaction between a donor and an acceptor ion. The model consists of a transferring proton with a mass of $m_{\mathrm{p}}$ and charge $z_{\mathrm{p}}$, an electron, and two fixed ions (a donor and an acceptor ion, with the charge of $z_{\mathrm{D}}$ and $z_{\mathrm{A}}$, respectively). The molecular Hamiltonian is $\hat{H}_{\mathrm{M}}=\frac{\hat{P}_{R}^{2}}{2 M}+\hat{H}_{\mathrm{el}}$, where $M$ is the mass of the nuclei (proton in this model), $\hat{H}_{\mathrm{el}}=\hat{T}_{r}+\hat{V}_{\mathrm{eN}}+\hat{V}_{\mathrm{NN}}$ is the electronic Hamiltonian, where $\hat{T}_{r}=\hat{p}_{\mathrm{r}}^{2} / 2 m_{\mathrm{e}}$ represents the kinetic energy operator of the electron with mass $m_{\mathrm{e}}$, $\hat{V}_{\mathrm{eN}}$ describes the interaction between the electron and the three nuclei, which is written as a modified Coulomb potential

$$
\hat{V}_{\mathrm{eN}}=-z_{\mathrm{p}} e^{2} \frac{\operatorname{erf}\left(\frac{|r-R|}{R_{\mathrm{c}}}\right)}{|r-R|}-\sum_{\alpha \in \mathrm{D}, \mathrm{A}} z_{\alpha} e^{2} \frac{\operatorname{erf}\left(\frac{\left|r-R_{\alpha}\right|}{R_{\mathrm{c}}}\right)}{\left|r-R_{\alpha}\right|},
$$

where $r$ is the position of the electron and $e=1$ a.u. is the fundamental charge, $R$ is the position of the proton, while $R_{\mathrm{D}}$ and $R_{\mathrm{A}}$ are the positions of the donor and acceptor ion, respectively. $R_{\mathrm{c}}$ is a parameter that controls the strength of the modified Coulomb potentials. The nucleus-nucleus interaction potential $V_{\mathrm{NN}}$ that describes the Coulomb repulsion between the proton and the static ions takes the form of

$$
V_{\mathrm{NN}}=\frac{z_{\mathrm{p}} z_{\mathrm{D}} e^{2}}{\left|R-R_{\mathrm{D}}\right|}+\frac{z_{\mathrm{p}} z_{\mathrm{A}} e^{2}}{\left|R-R_{\mathrm{A}}\right|}
$$

The parameters in the molecular Hamiltonian $\hat{H}_{\mathrm{M}}$ used in this work is tabulated in Table. I. The resulting PES $E(R)=\left\langle\Psi_{g}(R)\left|\left(\hat{H}_{\mathrm{M}}-\hat{T}_{R}\right)\right| \Psi_{g}(R)\right\rangle$ and the permanent dipole moment $\mu(R)=\left\langle\Psi_{g}(R)|\hat{\mu}| \Psi_{g}(R)\right\rangle$ are shown in Fig. 1b and Fig. 1c, respectively. The adiabatic ground state $\left|\Psi_{g}(R)\right\rangle$ is obtained using Fourier Grid Hamiltonian (FGH) approach [61, 62]. In particular, we use a total of $N=2000$ grid basis $\left\{\left|r_{i}\right\rangle\right\}$ to describe the electronic degrees of freedom $r$ in the range of $\left[R_{\mathrm{D}}-10, R_{\mathrm{A}}+10\right]$ (with $\Delta r=0.01$ a.u.). Details of this calculation is provided in the Supporting Information. 


\begin{tabular}{lc}
\hline \hline Parameter & Value (unit) \\
\hline$z_{\mathrm{p}}, z_{\mathrm{D}}, z_{\mathrm{A}}$ & 1 (unitless) \\
$R_{\mathrm{D}}$ & $-2.5(\AA)$ \\
$R_{\mathrm{A}}$ & $2.5(\AA)$ \\
$R_{\mathrm{c}}$ & $0.8(\AA)$ \\
$m_{\mathrm{p}}$ & 1836 (a.u.) \\
$m_{\mathrm{e}}$ & 1 (a.u.) \\
\hline \hline
\end{tabular}

TABLE I. Parameters used in the molecular Hamiltonian $\hat{H}_{\mathrm{M}}$.

Grote-Hynes Rate Theory. In multi-dimensional transition state theory, the reactant to product rate constant is given as $[39,63-65]$

$$
k=\frac{1}{2 \pi} \frac{\prod_{i=1}^{N} \tilde{\omega}_{i}^{0}}{\prod_{i=2}^{N} \tilde{\omega}_{i}^{\ddagger}} e^{-\beta E_{b}},
$$

where, $\left\{\tilde{\omega}_{i}\right\}$ are normal mode frequencies of the Hamiltonian in the reactant well, and $\left\{\tilde{\omega}_{2}^{\ddagger}, \ldots, \tilde{\omega}_{N}^{\ddagger}\right\}$ are the stable normal mode frequencies at the barrier, such that $\tilde{\omega}_{i}^{\ddagger 2}>0$ for $i>1$, and $\tilde{\omega}_{1}^{\ddagger 2}<0$ is the imaginary frequency of the transition state.

Considering a simplified model of the molecule-cavity hybrid system, $\hat{H}-\hat{H}_{\mathrm{vib}}=\frac{\hat{P}^{2}}{2 M}+E(R)+\frac{\hat{p}_{\mathrm{c}}^{2}}{2}+\frac{1}{2} \omega_{\mathrm{c}}^{2}\left(\hat{q}_{\mathrm{c}}+\right.$ $\left.\sqrt{\frac{2}{\hbar \omega_{\mathrm{c}}^{3}}} \chi \cdot \mu(R)\right)^{2}$ which only contains two DOFs $\left\{q_{\mathrm{c}}, R\right\}$, the normal modes frequencies at $R_{0}$ are $\tilde{\omega}_{ \pm}^{2}=\frac{1}{2}\left(\omega_{0}^{2}+\frac{\mathcal{C}_{0}^{2}}{\omega_{c}^{2}}+\right.$ $\left.\omega_{\mathrm{c}}^{2}\right) \pm \frac{1}{2} \sqrt{\left(\omega_{0}^{2}+\frac{\mathcal{C}_{0}^{2}}{\omega_{\mathrm{c}}^{2}}-\omega_{\mathrm{c}}^{2}\right)^{2}-4 \mathcal{C}_{0}^{2}}$, where $\mathcal{C}_{0}=\sqrt{\frac{2 \omega_{\mathrm{c}}}{M}} \chi \cdot \mu_{0}^{\prime}$. Note that they can also be equivalently expressed as $\tilde{\omega}_{ \pm}^{2}=\frac{1}{2}\left(\omega_{0}^{2}+\frac{\mathcal{C}_{0}^{2}}{\omega_{\mathrm{c}}^{2}}+\omega_{\mathrm{c}}^{2}\right) \pm \frac{1}{2} \sqrt{\left(\omega_{0}^{2}+\frac{\mathcal{C}_{0}^{2}}{\omega_{\mathrm{c}}^{2}}+\omega_{\mathrm{c}}^{2}\right)^{2}-4 \omega_{\mathrm{c}}^{2} \omega_{0}^{2}}$. The normal mode frequencies at $R_{\ddagger}$ are $\tilde{\omega}_{ \pm}^{\ddagger 2}=\frac{1}{2}\left(-\omega_{\mathrm{b}}^{2}+\right.$ $\left.\frac{\mathcal{C}_{\ddagger}^{2}}{\omega_{\mathrm{c}}^{2}}+\omega_{\mathrm{c}}^{2}\right) \pm \frac{1}{2} \sqrt{\left(-\omega_{\mathrm{b}}^{2}+\frac{\mathcal{C}_{\ddagger}^{2}}{\omega_{\mathrm{c}}^{2}}+\omega_{\mathrm{c}}^{2}\right)^{2}+4 \omega_{\mathrm{c}}^{2} \omega_{\mathrm{b}}^{2}}$, where $\mathcal{C}_{\ddagger}=$ $\sqrt{\frac{2 \omega_{c}}{M}} \chi \cdot \mu_{\ddagger}^{\prime}$. Using these normal mode frequencies, the rate constant in Eq. 8 for the $\hat{H}-\hat{H}_{\text {vib }}$ model is expressed as $k=\frac{1}{2 \pi} \frac{\tilde{\omega}_{+} \tilde{\omega}_{-}}{\tilde{\omega}_{+}^{\ddagger}} e^{-\beta E_{b}}$, where $E_{\mathrm{b}}$ is the energy barrier. Using the fact that $\left(\tilde{\omega}_{+}^{\ddagger} \tilde{\omega}_{-}^{\ddagger}\right)^{2}=-\omega_{\mathrm{b}}^{2} \omega_{\mathrm{c}}^{2}$ and $\left(\tilde{\omega}_{+} \tilde{\omega}_{-}\right)^{2}=\omega_{0}^{2} \omega_{\mathrm{c}}^{2}$, the rate constant can be further expressed as follows

$k=\frac{1}{2 \pi} \sqrt{-\left(\omega_{-}^{\ddagger}\right)^{2}} \frac{\omega_{0} \omega_{\mathrm{c}}}{\omega_{\mathrm{b}} \omega_{\mathrm{c}}} e^{-\beta E_{b}}=\frac{\lambda}{\omega_{\mathrm{b}}} \cdot \frac{\omega_{0}}{2 \pi} e^{-\beta E_{b}} \equiv \kappa_{\mathrm{GH}} \cdot k_{\mathrm{TST}}$,

where $k_{\mathrm{TST}}=\frac{\omega_{0}}{2 \pi} e^{-\beta E_{\mathrm{b}}}, \kappa_{\mathrm{GH}}=\frac{\lambda}{\omega_{\mathrm{b}}}$ is the transmission coefficient in the GH theory, $\lambda=\sqrt{-\left(\omega_{-}^{\ddagger}\right)^{2}}$, and $\kappa_{\mathrm{GH}}=$ $\frac{\lambda}{\omega_{\mathrm{b}}}$, which is Eq 5 .

Alternatively, one can derive the transmission coefficient $\kappa_{\mathrm{GH}}$ from the equation of motion [38, 52]. It is straightforward to derive (see SI) the generalized Langevin equation (GLE) of $R$ governed by $\hat{H}-\hat{H}_{\mathrm{vib}}$ as $\ddot{R}=\omega_{\mathrm{b}}^{2} R(t)-\int_{0}^{t} \gamma(t-\tau) \dot{R}(t) d \tau+\xi(t)$, where $\gamma(t)=$ $\frac{\mathcal{C}_{f}^{2}}{\omega_{c}^{2}} \cos \left(\omega_{\mathrm{c}} t\right)$ is the the friction kernel and the random force obeys $\langle\xi(0) \xi(t)\rangle=k_{\mathrm{B}} T \cdot \gamma(t)$. Using $R(t)=B_{0} e^{\lambda t}$ as a solution for a reactive trajectory [38], performing an ensemble average of the above GLE, using the fact $\langle\xi(t)\rangle=0$, and taking the limit $t \rightarrow \infty$, we obtain $\lambda^{2}=\omega_{\mathrm{b}}^{2}-\lambda \int_{0}^{\infty} \gamma(\tau) e^{\lambda \tau} d \tau=\omega_{\mathrm{b}}^{2}-\lambda \tilde{\gamma}(\lambda)$, where $\tilde{\gamma}(\lambda)=\int_{0}^{\infty} \gamma(\tau) e^{\lambda \tau} d \tau=\frac{\mathcal{C}_{f}^{2}}{\omega_{c}^{2}} \frac{\lambda}{\lambda^{2}+\omega_{c}^{2}}$ is the Laplace transformed friction kernel $\gamma(t)$. This leads to the following equation that $\lambda$ satisfies

$$
\lambda^{4}+\left[\omega_{\mathrm{c}}^{2}-\omega_{\mathrm{b}}^{2}+2 \frac{\left(\chi \mu_{\ddagger}^{\prime}\right)^{2}}{M \omega_{\mathrm{c}}}\right] \lambda^{2}-\omega_{\mathrm{c}}^{2} \omega_{\mathrm{b}}^{2}=0,
$$

whose solution leads to the same expression for $\kappa_{\mathrm{GH}}=\frac{\lambda}{\omega_{\mathrm{b}}}$ in Eq. 5. When explicitly considering the dissipative environment $\hat{H}_{\text {vib }}$ in Eq. 1, the above procedure for deriving $\kappa_{\mathrm{GH}}$ can be followed, and the detailed derivation is provided in the Supporting Information.

Numerical Calculations of $\kappa(t)$. To compute $\kappa(t)$ from the flux-side correlation function in Eq. 4, the photon mode $q_{\mathrm{c}}$ and the molecular reaction coordinate $R$ was treated classically, and the influence of $\hat{H}_{\text {vib }}$ on $R$ is simulated through Langevin dynamics (with the details provided in the Supporting Information). All simulations were performed under $T=300 \mathrm{~K}$. The time step $\Delta t$ we chose was 4 a.u., which was carefully checked to produce stable integration for all simulations. From a constraint MD trajectory with $R_{\ddagger}=0$ and the Langevin dynamics, the constrained configurations $\left\{q_{c}, R_{\ddagger}\right\}$ are sampled for every 270 fs along the constrained trajectory. A total of 100,000 trajectories are released from the dividing surface, with the velocity randomly sampled from the classical Maxwell-Boltzmann distribution. Each of the child trajectory was propagated for $200 \mathrm{fs}$, which guaranteed that the flux-side correlation function would plateau. Langevin dynamics was applied to the molecular DOF in this process to dissipate the excess energy to ensure a plateau in the correlation function. The friction parameter $\gamma$ was chosen to be $400 \mathrm{~cm}^{-1}$, according to the spectral density of the $\hat{H}_{\text {vib }}$.

Effective $\Delta\left(\Delta G^{\ddagger}\right)$. To account the "effective change" of the Gibbs free energy barrier $\Delta\left(\Delta G^{\ddagger}\right)$ that is equivalent to the change of $\kappa$, we consider the Eyring rate equation $k=\frac{k_{\mathrm{B}} T}{h} e^{-\frac{\Delta G^{\ddagger}}{k_{\mathrm{B}} T}}$, and thus $\Delta G^{\ddagger}=-\frac{1}{\beta} \ln (2 \pi \beta \cdot k)$. With $k=\kappa \cdot k_{\mathrm{TST}}$, we can rewrite the above $\Delta G^{\ddagger}$ as

$$
\Delta G^{\ddagger}=-\frac{1}{\beta} \ln \kappa-\frac{1}{\beta} \ln 2 \pi \beta k_{\mathrm{TST}}
$$

Because $k_{\text {TST }}$ is a constant at any coupling strength and cavity frequency and is same for bare molecular case. The effective $\Delta\left(\Delta G^{\ddagger}\right)$ solely depends on the change of $\kappa$. The change of free energy barrier compared to the bare molecular reaction (with a $\kappa_{0}$ and $\delta G_{0}^{\ddagger}$ ) is then $\Delta\left(\Delta G^{\ddagger}\right)=\Delta G^{\ddagger}-\Delta G_{0}^{\ddagger}=-\frac{1}{\beta} \ln \frac{\kappa}{\kappa_{0}}$.

Absorption Spectrum. We employ a simple scheme [66] to compute the absorption spectrum of the 
molecule-cavity hybrid system. We assume that the external light source is only coupled to molecular transition dipoles [66] and we introduce a phenomenological width $\varepsilon$ to account for the broadening of the absorption spectrum. With these simplifications the absorption spectrum can be obtained analytically. The absorption cross section $\sigma(E)$ as a function of excitation energy $E$ is expressed [66, 67] as follows

$$
\sigma(E)=\frac{4 \pi E}{c} \operatorname{Im}\left[\sum_{k \neq 0} \frac{\left|\left\langle\psi_{k}|\hat{\mu}| \psi_{0}\right\rangle\right|^{2}}{E_{k}-E_{0}-E-i \varepsilon}\right]
$$

where $E_{k}$ is the $k_{\text {th }}$ polaritonic eigenenergy obtained by solving the eigenstate of polariton Hamiltonian $\hat{H}_{\mathrm{pl}}=$ $\hat{H}-\hat{T}_{R}-\hat{H}_{\text {vib }}$ with the basis $\left\{\left|\Psi_{0}\right\rangle \otimes|n\rangle\right\}$, where $|n\rangle$ is the Fock states of the vacuum. We have used 100 Fock states to ensure convergence of the polariton eigenspectrum for the IR spectroscopy calculations.

\section{ACKNOWLEDGMENT}

This work was supported by the National Science Foundation CAREER Award under Grant No. CHE1845747 and "Enabling Quantum Leap in Chemistry" program under a Grant number CHE-1836546, as well as by a Cottrell Scholar award (a program by of Research Corporation for Science Advancement). Computing resources were provided by the Center for Integrated Research Computing (CIRC) at the University of Rochester.

* amandal4@ur.rochester.edu

† pengfei.huo@rochester.edu

[1] T. W. Ebbesen, Hybrid light-matter states in a molecular and material science perspective, Acc. Chem. Res. 49, 2403 (2016).

[2] R. F. Ribeiro, L. A. Martínez-Martínez, M. Du, J. Campos-Gonzalez-Angulo, and J. Yuen-Zhou, Polariton chemistry: Controlling molecular dynamics with optical cavities, Chem. Sci. 9, 6325 (2018).

[3] J. Feist, J. Galego, and F. J. Garcia-Vidal, Polaritonic chemistry with organic molecules, ACS Photonics 5, 205 (2018).

[4] F. Herrera and J. Owrutsky, Molecular polaritons for controlling chemistry with quantum optics, J. Chem. Phys. 152, 100902 (2020).

[5] K. Hirai, J. A. Hutchison, and H. Uji-i, Recent progress of vibropolaritonic chemistry, ChemPlusChem (2020).

[6] J. A. Hutchison, T. Schwartz, C. Genet, E. Devaux, and T. W. Ebbesen, Modifying chemical landscapes by coupling to vacuum fields, Angew. Chem. Int. Ed. 51, 1592 (2012).

[7] J. Galego, F. J. Garcia-Vidal, and J. Feist, Suppressing photochemical reactions with quantized light fields, Nat. Commun. 7, 13841 EP (2016).
[8] J. Galego, F. J. Garcia-Vidal, and J. Feist, Manymolecule reaction triggered by a single photon in polaritonic chemistry, Phys. Rev. Lett. 119, 136001 (2017).

[9] A. Mandal and P. Huo, Investigating new reactivities enabled by polariton photochemistry, J. Phys. Chem. Lett. 10, 5519 (2019).

[10] F. Herrera and F. C. Spano, Cavity-controlled chemistry in molecular ensembles, Phys. Rev. Lett. 116, 238301 (2016).

[11] A. Semenov and A. Nitzan, Electron transfer in confined electromagnetic fields, J. Chem. Phys. 150, 174122 (2019).

[12] A. Mandal, T. D. Krauss, and P. Huo, Polaritonmediated electron transfer via cavity quantum electrodynamics, J. Phys. Chem. B 124, 6321 (2020).

[13] M. Du, R. F. Ribeiro, and J. Yuen-Zhou, Remote control of chemistry in optical cavities, Chem 5, 1167 (2019).

[14] J. George, T. Chervy, A. Shalabney, E. Devaux, H. Hiura, C. Genet, and T. Ebbesen, Multiple rabi splittings under ultra-strong vibrational coupling, Phys. Rev. Lett. 117, 153601 (2016).

[15] A. Shalabney, J. George, J. Hutchison, G. Pupillo, C. Genet, and T. W. Ebbesen, Coherent coupling of molecular resonators with a microcavity mode, Nat. Commun. 6, 5981 (2015).

[16] L. Martínez-Martínez, R. Ribeiro, J. Campos-GonzálezAngulo, and J. Yuen-Zhou, Can ultrastrong coupling change ground state chemical reactions?, ACS Photonics 5, 167 (2018).

[17] J. Galego, C. Climent, F. J. Garcia-Vidal, and J. Feist, Cavity casimir-polder forces and their effects in groundstate chemical reactivity, Phys. Rev. X 9, 021057 (2019).

[18] T. E. Li, A. Nitzan, and J. E. Subotnik, On the origin of ground-state vacuum-field catalysis: Equilibrium consideration, J. Chem. Phys. 152, 234107 (2020).

[19] J. A. Campos-Gonzalez-Angulo and J. Yuen-Zhou, Polaritonic normal modes in transition state theory, J. Chem. Phys. 152, 161101 (2020).

[20] A. Thomas, J. George, A. Shalabney, M. Dryzhakov, S. J. Varma, J. Moran, T. Chervy, X. Zhong, E. Devaux, C. Genet, J. A. Hutchison, and T. W. Ebbesen, Ground-state chemical reactivity under vibrational coupling to the vacuum electromagnetic field, Angew. Chem. 128, 11634 (2016).

[21] A. Thomas, L. Lethuillier-Karl, K. Nagarajan, R. M. A. Vergauwe, J. George, T. Chervy, A. Shalabney, E. Devaux, C. Genet, J. Moran, and T. W. Ebbesen, Tilting a ground-state reactivity landscape by vibrational strong coupling, Science 363, 615 (2019).

[22] A. Thomas, A. Jayachandran, L. Lethuillier-Karl, R. M. Vergauwe, K. Nagarajan, E. Devaux, C. Genet, J. Moran, and T. W. Ebbesen, Ground state chemistry under vibrational strong coupling: dependence of thermodynamic parameters on the rabi splitting energy, Nanophotonics 9 (2020).

[23] R. M. A. Vergauwe, A. Thomas, K. Nagarajan, A. Shalabney, J. George, T. Chervy, M. Seidel, E. Devaux, V. Torbeev, and T. W. Ebbesen, Modification of enzyme activity by vibrational strong coupling of water, Angew. Chem. Int. Ed. 58, 15324 (2019).

[24] K. Hirai, R. Takeda, J. A. Hutchison, and H. Uji-i, Modulation of prins cyclization by vibrational strong coupling, Angew. Chem. Int. Ed. 59, 5332 (2020). 
[25] J. Lather, P. Bhatt, A. Thomas, T. W. Ebbesen, and J. George, Cavity catalysis by cooperative vibrational strong coupling of reactant and solvent molecules, Angew. Chem. Int. Ed. 58, 10635 (2019).

[26] H. Hiura, A. Shalabney, and J. George, Cavity catalysisaccelerating reactions under vibrational strong coupling, ChemRxiv (2019).

[27] V. P. Zhdanov, Vacuum field in a cavity, light-mediated vibrational coupling, and chemical reactivity, Chem. Phys. 535, 110767 (2020).

[28] J. A. Campos-Gonzalez-Angulo, R. F. Ribeiro, and J. Yuen-Zhou, Resonant catalysis of thermally activated chemical reactions with vibrational polaritons, Nature Communications 10, 4685 (2019).

[29] R. F. Grote and J. T. Hynes, The stable states picture of chemical reactions. ii. rate constants for condensed and gas phase reaction models, J. Chem. Phys. 73, 2715 (1980).

[30] G. Ciccotti, M. Ferrario, J. T. Hynes, and R. Kapral, Dynamics of ion pair interconversion in a polar solvent, J. Chem. Phys. 93, 7137 (1990).

[31] J. Flick, M. Ruggenthaler, H. Appel, and A. Rubio, Atoms and molecules in cavities, from weak to strong coupling in quantum-electrodynamics (qed) chemistry, Proc. Natl. Acad. Sci. U. S. A. 114, 3026 (2017).

[32] O. Vendrell, Coherent dynamics in cavity femtochemistry: Application of the multi-configuration timedependent hartree method, Chem. Phys. 509, 55 (2018).

[33] C. Schäfer, M. Ruggenthaler, and A. Rubio, Ab initio nonrelativistic quantum electrodynamics: Bridging quantum chemistry and quantum optics from weak to strong coupling, Phys. Rev. A 98, 043801 (2018).

[34] A. Frisk Kockum, A. Miranowicz, S. De Liberato, S. Savasta, and F. Nori, Ultrastrong coupling between light and matter, Nat. Rev. Phys. 1, 19 (2019).

[35] L. J. Flick, H. Appel, M. Ruggenthaler, and A. Rubio, Cavity born-oppenheimer approximation for correlated electron-nuclear-photon systems, J. Chem. Theory Comput. 13, 1616 (2017).

[36] D. Frenkel and B. Smit, Understanding Molecular Simulation (Elsevier, 2002).

[37] W. H. Miller, S. D. Schwartz, and J. W. Tromp, Quantum mechanical rate constants for bimolecular reactions, J. Chem. Phys. 79, 4889 (1983).

[38] B. J. Gertner, K. R. Wilson, and J. T. Hynes, Nonequilibrium solvation effects on reaction rates for model sn2 reactions in water, J. Chem. Phys. 90, 3537 (1989).

[39] P. Hänggi, P. Talkner, and M. Borkovec, Reaction-rate theory: fifty years after kramers, Rev. Mod. Phys. 62, 251 (1990).

[40] P. Hanggi and F. Mojtabai, Thermally activated escape rate in presence of long-time memory, Phys. Rev. A 26, 1168 (1982).

[41] B. Carmeli and A. Nitzan, Non-markovian theory of activated rate processes. iii. bridging between the kramers limits, Phys. Rev. A 29, 1481 (1984).

[42] S. C. Tucker, M. E. Tuckerman, B. J. Berne, and E. Pollak, Comparison of rate theories for generalized langevin dynamics, J. Chem. Phys 95, 5809 (1991).

[43] J. P. Bergsma, B. J. Gertner, K. R. Wilson, and J. T. Hynes, Molecular dynamics of a model sn2 reaction in water, J. Chem. Phys. 86, 1356 (1987).

[44] W. P. Keirstead, K. R. Wilson, and J. T. Hynes, Molecular dynamics of a model sn1 reaction in water, J. Chem.
Phys. 95, 5256 (1991).

[45] I. S. Tolokh, G. W. N. White, S. Goldman, and C. G. Gray, Prediction of ion channel transport from grotehynes and kramers theories, Mol. Phys. 100, 2351 (2002).

[46] M. Roca, V. Moliner, I. Tuñón, and J. T. Hynes, Coupling between protein and reaction dynamics in enzymatic processes: Application of grote-hynes theory to catechol o-methyltransferase, J. Am. Chem. Soc. 128, 6186 (2006).

[47] N. Kanaan, M. Roca, I. Tunon, S. Marti, and V. Moliner, Application of grote-hynes theory to the reaction catalyzed by thymidylate synthase, J. Phys. Chem. B 114, 13593 (2010).

[48] H. Hiura, A. Shalabney, and J. George, A reaction kinetic model for vacuum-field catalysis based on vibrational light-matter coupling, ChemRxiv (2019).

[49] B. Peters, Reaction rate theory and rare event, Elsevier (2017).

[50] G. van der Zwan and J. Hynes, Nonequilibrium solvation dynamics in solution reactionsa, J. Chem. Phys. 78, 4174-4185 (1983).

[51] G. van der Zwan and J. T. Hynes, Dynamical polar solvent effects on solution reactions: A simple continuum model, J. Chem. Phys 76, 2993 (1982).

[52] N. E. Henriksen and F. Y. Hansen, Theories of molecular reaction dynamics: the microscopic foundation of chemical kinetics, Oxford University Press (2008).

[53] A. Thomas, L. Lethuillier-Karl, K. Nagarajan, R. M. A. Vergauwe, J. George, T. Chervy, A. Shalabney, E. Devaux, C. Genet, J. Moran, and T. W. Ebbesen, Tilting a ground-state reactivity landscape by vibrational strong coupling, Science 363, 615 (2019).

[54] D. G. Truhlar and B. C. Garrett, Multidimensional transition state theory and the validity of grote-hynes theory, J. Phys. Chem. B 104, 1069 (2000).

[55] E. A. Power and S. Zienau, Coulomb gauge in nonrelativistic quantum electro-dynamics and the shape of spectral lines, Philosophical Transactions of the Royal Society of London A, Mathematical and Physical Sciences 251, 427 (1959).

[56] C. Cohen-Tannoudji, J. Dupont-Roc, and G. Grynberg, Photons and atoms: Introduction to quantum electrodynamics, John Wiley \& Sons, Inc. (1989).

[57] V. Rokaj, D. M. Welakuh, M. Ruggenthaler, and A. Rubio, Light-matter interaction in the longwavelength limit: no ground-state without dipole self-energy, J. Phys. B: At. Mol. Opt. Phys. 51, 034005 (2018).

[58] C. Schäfer, M. Ruggenthaler, V. Rokaj, and A. Rubio, Relevance of the quadratic diamagnetic and selfpolarization terms in cavity quantum electrodynamics, ACS Photonics 7, 975 (2020).

[59] D. D. Bernardis, P. Pilar, T. Jaako, S. D. Liberato, and P. Rabl, Breakdown of gauge invariance in ultrastrongcoupling cavity qed, Phys. Rev. A 98, 053819 (2018).

[60] S. Shin and H. Metiu, Nonadiabatic Effects on the Charge Transfer Rate Constant: A Numerical Study of a Simple Model System, J. Chem. Phys. 102, 9285 (1995).

[61] C. C. Marston and G. G. Balint-Kurti, The fourier grid hamiltonian method for bound state eigenvalues and eigenfunctions, J. Chem. Phys. 91, 3571 (1989).

[62] D. Tannor, Introduction to quantum mechanics: A timedependent perspective, University Science books (2007).

[63] H. Eyring, The activated complex in chemical reactions, J. Chem. Phys. 3, 107 (1935). 
[64] N. B. Slater, New formulation of gaseous unimolecular dissociation rates, J. Chem. Phys. 24, 1256 (1956).

[65] E. Pollak, Theory of activated rate processes: A new derivation of kramers' expression, J. Chem. Phys. 85, 865 (1986).
[66] J. Galego, F. J. Garcia-Vidal, and J. Feist, Cavityinduced modifications of molecular structure in the strong-coupling regime, Phys. Rev. X 5, 041022 (2015).

[67] T. N. Rescigno and V. McKoy, Rigorous method for computing photoabsorption cross sections from a basis-set expansion, Phys. Rev. A 12, 522 (1975). 